Research Article

\title{
Sampled-Data-Based Adaptive Group Synchronization of Second-Order Nonlinear Complex Dynamical Networks with Time-Varying Delays
}

\author{
Bo Liu $\mathbb{D}^{1},{ }^{1}$ Jiahui Bai, ${ }^{2}$ Yue Zhao, ${ }^{1}$ Chao Liu $\mathbb{D},{ }^{3}$ Xuemin Yan, ${ }^{4}$ and Hongtao Zhou ${ }^{5}{ }^{5}$ \\ ${ }^{1}$ School of Information Engineering, Minzu University of China, Beijing 100081, China \\ ${ }^{2}$ College of Science, North China University of Technology, Beijing 100144, China \\ ${ }^{3}$ School of Mathematics, Shanghai University of Finance and Economics, Shanghai 200433, China \\ ${ }^{4}$ National Institute of Education Sciences, Beijing 100088, China \\ ${ }^{5}$ Key Laboratory of Imaging Processing and Intelligence Control, School of Artificial Intelligence and Automation, \\ Huazhong University of Science and Technology, Wuhan 430074, China
}

Correspondence should be addressed to Bo Liu; boliu@ncut.edu.cn

Received 23 April 2020; Accepted 5 June 2020; Published 27 June 2020

Academic Editor: Zhiwei Gao

Copyright $\odot 2020$ Bo Liu et al. This is an open access article distributed under the Creative Commons Attribution License, which permits unrestricted use, distribution, and reproduction in any medium, provided the original work is properly cited.

This paper studies the adaptive group synchronization of second-order nonlinear complex dynamical networks with sampled-data and time-varying delays by designing a new adaptive strategy to feedback gains and coupling strengths. According to Lyapunov stability properties, it is shown that the agents of subgroups can converge the given synchronous states, respectively, under some conditions on the sampled period. Moreover, some simulation results are given.

\section{Introduction}

Complex dynamical networks are used to describe the large size and complexity of the research object to solve the practical application problem by constructing the mathematical models in essence. In nature, synchronization is a ubiquitous phenomenon, such as the synchronization of beating rhythm of cardiac myocytes and consistency of fireflies twinkling. Recently, the synchronization problem of complex systems with nonlinear dynamical has attracted increasing attention and wide application including physics, mathematics, chemistry, biology, information science, electronics, and medicine [1-16]. Because of the extensive application value of synchronization in engineering technology, complex network synchronization has become a hot issue in the field of nonlinearity science, for example, the evolutionary origin of asymptotically stable consensus in [7] and the application of synchronization in engineering was introduced in [8].

In order to achieve network synchronization, some advisable methods are introduced in outstanding works (e.g., [17-28]), such as pinning control [17-19] and adaptive strategies [20-28]. In [25], the authors introduced the adaptive coupling strengths and studied the adaptive synchronization of two heterogeneous second-order nonlinear coupled dynamical systems. The synchronization of fractional-order complex networks were well considered in [26-28] and applying decentralized adaptive strategies, pinning control and adaptive control strategy, respectively. The authors [29-31] investigated the synchronization of complex dynamical systems with time-varying delays. Works [32, 33] discussed adaptive consensus of networks with single-integrator nonlinear dynamics and adaptive synchronization of networks with double-integrator nonlinear dynamics, respectively. In [34], the author investigated the adaptive synchronization for first-order complex systems with local Lipschitz nonlinearity. Su et al. [35] also researched the adaptive flocking of multiagent networks with local Lipschitz nonlinearity. In engineering practice, the whole network (group) can be partitioned into several subnetworks (subgroups) to study the synchronization 
problems, called as group synchronization. Li et al. [36] investigated the group synchronization for complex systems with nonlinear dynamics. Some conditions were established in [37] for solving consensus problem of multiagent complex systems with double-integrator and sampled control. The consensus of complex networks with sampled data and timedelay topology was studied in [38].

Inspired by these works, the adaptive group synchronization of second-order nonlinear complex dynamical undirected networks with sampled-data and time-varying delays will be discussed in this paper. And its main contributions are threefold: (1) the new second-order model with sampled-data and time-varying delays is established; (2) the communication delays of all the neighboring agents' positions and velocities are time varying; (3) adaptive laws for solving the group synchronization of second-order nonlinear complex dynamical systems are introduced.

The rest of this paper is arranged as follows. The mathematical model with time delays and sampled data and some necessary preliminaries are given in Section 2. Section 3 presents the main results. Some numerical simulations are given in Section 4. Finally, Section 5 shows the conclusion.

\section{Problem Formulation and Preliminaries}

A second-order complex network with nonlinear dynamics consists of $N$ nodes and each node obeys

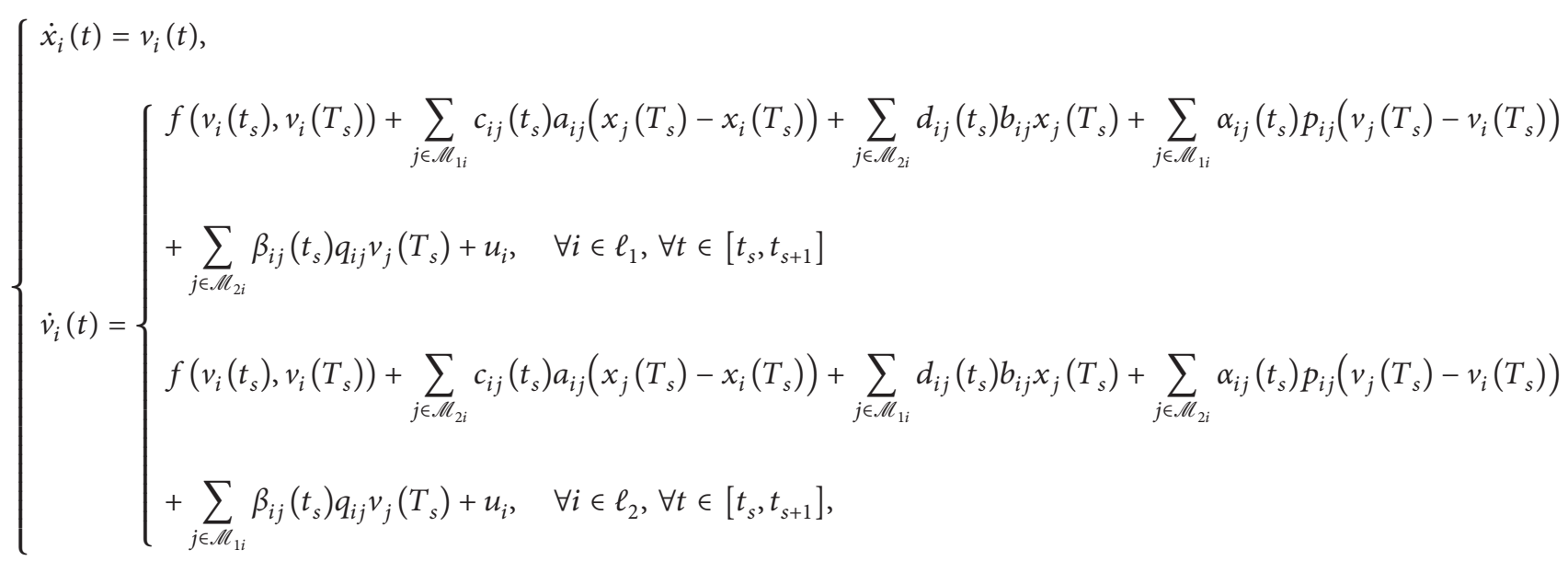

where $x_{i}(t) \in R^{n}$ is the position vector of agent $i ; v_{i}(t) \in R^{n}$ is its velocity vector, for $i=1, \ldots, N$ as $t \in[0,+\infty)$; $f: R^{n} \longrightarrow R^{n}$ is a continuous differentiable function; $T_{s}=$ $t_{s}-\tau\left(t_{s}\right)$ and $\tau>0 ; \mathscr{M}_{i}$ is the neighbor set of node $i$, $\mathscr{M}_{i} \in \mathscr{M}_{1 i} \cup \mathscr{M}_{2 i}$ with $\mathscr{M}_{1 i}=\left\{x_{j} \in \mathscr{X}_{1}: a_{i j} \geq 0, i, j \in \ell_{1}\right\}$ and $\mathscr{M}_{2 i}=\left\{x_{j} \in \mathscr{X}_{2}: a_{i j} \geq 0, i, j \in \ell_{2}\right\}$, where $\mathscr{X}=\mathscr{X}_{1} \cup \mathscr{X}_{2}, \ell=$ $\ell_{1} \cup \ell_{2}$ with $\mathscr{X}_{1}=\left\{x_{1}, \ldots, x_{L}\right\}, \mathscr{X}_{2}=\left\{x_{L+1}, \ldots, x_{N}\right\}, \ell_{1}=$
$1, \ldots, L, \quad \ell_{2}=L+1, \ldots, N, \quad$ and $\quad L<N ; \quad c_{i j}\left(t_{s}\right), d_{i j}\left(t_{s}\right)$, $\alpha_{i j}\left(t_{s}\right)$, and $\beta_{i j}\left(t_{s}\right)$ are the position's and velocity's coupling strengths between agent $i$ and agent $j$; and nonnegative numbers $a_{i j}, b_{i j}, p_{i j}$, and $q_{i j}$ are the edge-weights connecting agent $i$ and agent $j$.

Design the control input as

$$
u_{i}= \begin{cases}-c_{i}\left(t_{s}\right) h_{i}\left(x_{i}\left(T_{s}\right)-\bar{x}_{1}\left(T_{s}\right)\right)-d_{i}\left(t_{s}\right) l_{i}\left(v_{i}\left(T_{s}\right)-\bar{v}_{1}\left(T_{s}\right)\right), & i \in \ell_{1}, \\ -c_{i}\left(\left(t_{s}\right)\right) h_{i}\left(x_{i}\left(T_{s}\right)-\bar{x}_{2}\left(T_{s}\right)\right)-d_{i}\left(t_{s}\right) l_{i}\left(v_{i}\left(T_{s}\right)-\bar{v}_{2}\left(T_{s}\right)\right), & i \in \ell_{2},\end{cases}
$$

where $h_{i}$ and $l_{i}$ are on-off controls, if node $i$ is steered, then $h_{i}=1$ and $l_{i}=1$, otherwise $h_{i}=0$ and $l_{i}=0, c_{i}\left(t_{s}\right)$ and $d_{i}\left(t_{s}\right)$ represent the position's and velocity's feedback gains, respectively, $\bar{x}_{1}(t) \in R^{n}$ and $\bar{x}_{2}(t) \in R^{n}$ are the given synchronous positions, and $\bar{v}_{1}(t) \in R^{n}$ and $\bar{v}_{2}(t) \in R^{n}$ are their velocities, respectively. 
According to (1) and (2), we design the adaptive laws for coupling strengths respectively as

$$
\begin{aligned}
& \dot{c}_{i j}\left(t_{s}\right)=\left\{\begin{array}{l}
a_{i j} k_{i j}\left[\left(x_{i}\left(T_{s}\right)-x_{j}\left(T_{s}\right)\right)^{T}\left(x_{i}\left(T_{s}\right)-x_{j}\left(T_{s}\right)\right)+\left(\dot{x}_{i}(t)-\dot{x}_{j}(t)\right)^{T}\left(\dot{x}_{i}(t)-\dot{x}_{j}(t)\right)\right], \quad i, j \in \ell_{1}, \\
a_{i j} k_{i j}\left[\left(x_{i}\left(T_{s}\right)-x_{j}\left(T_{s}\right)\right)^{T}\left(x_{i}\left(T_{s}\right)-x_{j}\left(T_{s}\right)\right)+\left(\dot{x}_{i}(t)-\dot{x}_{j}(t)\right)^{T}\left(\dot{x}_{i}(t)-\dot{x}_{j}(t)\right)\right], \quad i, j \in \ell_{2},
\end{array}\right. \\
& \dot{\alpha}_{i j}\left(t_{s}\right)= \begin{cases}p_{i j} \varepsilon_{i j}\left[\left(v_{i}\left(T_{s}\right)-v_{j}\left(T_{s}\right)\right)^{T}\left(v_{i}\left(T_{s}\right)-v_{j}\left(T_{s}\right)\right)+\left(\dot{v}_{i}(t)-\dot{v}_{j}(t)\right)^{T}\left(\dot{v}_{i}(t)-\dot{v}_{j}(t)\right)\right], i, j \in \ell_{1}, \\
p_{i j} \varepsilon_{i j}\left[\left(v_{i}\left(T_{s}\right)-v_{j}\left(T_{s}\right)\right)^{T}\left(v_{i}\left(T_{s}\right)-v_{j}\left(T_{s}\right)\right)+\left(\dot{v}_{i}(t)-\dot{v}_{j}(t)\right)^{T}\left(\dot{v}_{i}(t)-\dot{v}_{j}(t)\right)\right], i, j \in \ell_{2},\end{cases} \\
& \dot{d}_{i j}\left(t_{s}\right)= \begin{cases}b_{i j} k_{i j}\left(x_{j}\left(T_{s}\right)-\bar{x}_{2}\left(T_{s}\right)\right)^{T}\left(x_{j}\left(T_{s}\right)-\bar{x}_{2}\left(T_{s}\right)\right), & i \in \ell_{1}, j \in \ell_{2}, \\
b_{i j} k_{i j}\left(x_{j}\left(T_{s}\right)-\bar{x}_{1}\left(T_{s}\right)\right)^{T}\left(x_{j}\left(T_{s}\right)-\bar{x}_{1}\left(T_{s}\right)\right), & i \in \ell_{2}, j \in \ell_{1},\end{cases} \\
& \dot{\beta}_{i j}\left(t_{s}\right)= \begin{cases}q_{i j} \varepsilon_{i j}\left(v_{j}\left(T_{s}\right)-\bar{v}_{2}\left(T_{s}\right)\right)^{T}\left(v_{j}\left(T_{s}\right)-\bar{v}_{2}\left(T_{s}\right)\right), & i \in \ell_{1}, j \in \ell_{2}, \\
q_{i j} \varepsilon_{i j}\left(v_{j}\left(T_{s}\right)-\bar{v}_{1}\left(T_{s}\right)\right)^{T}\left(v_{j}\left(T_{s}\right)-\bar{v}_{1}\left(T_{s}\right)\right), & i \in \ell_{2}, j \in \ell_{1} .\end{cases}
\end{aligned}
$$

where $k_{i j}>0$ and $\varepsilon_{i j}>0$ are the weights of $c_{i j}\left(t_{s}\right)$ and $\alpha_{i j}\left(t_{s}\right)$, respectively.
Similarly, we design the adaptive laws for the feedback gains, respectively, as

$$
\begin{aligned}
& \dot{c}_{i}\left(t_{s}\right)=\left\{\begin{array}{l}
h_{i} k_{i}\left[\left(x_{i}\left(T_{s}\right)-\bar{x}_{1}\left(T_{s}\right)\right)^{T}\left(x_{i}\left(T_{s}\right)-\bar{x}_{1}\left(T_{s}\right)\right)+\left(\dot{x}_{i}(t)-\dot{\bar{x}}_{1}(t)\right)^{T}\left(\dot{x}_{i}(t)-\dot{\bar{x}}_{1}(t)\right)\right], \quad i \in \ell_{1}, \\
h_{i} k_{i}\left[\left(x_{i}\left(T_{s}\right)-\bar{x}_{2}\left(T_{s}\right)\right)^{T}\left(x_{i}\left(T_{s}\right)-\bar{x}_{2}\left(T_{s}\right)\right)+\left(\dot{x}_{i}(t)-\dot{\bar{x}}_{2}(t)\right)^{T}\left(\dot{x}_{i}(t)-\dot{\bar{x}}_{2}(t)\right)\right], \quad i \in \ell_{2},
\end{array}\right. \\
& \dot{d}_{i}\left(t_{s}\right)=\left\{\begin{array}{l}
l_{i} \varepsilon_{i}\left[\left(v_{i}\left(T_{s}\right)-\bar{v}_{1}\left(T_{s}\right)\right)^{T}\left(v_{i}\left(T_{s}\right)-\bar{v}_{1}\left(T_{s}\right)\right)+\left(\dot{v}_{i}(t)-\dot{\bar{v}}_{1}(t)\right)^{T}\left(\dot{v}_{i}(t)-\dot{\bar{v}}_{1}(t)\right)\right], \quad i \in \ell_{1}, \\
l_{i} \varepsilon_{i}\left[\left(v_{i}\left(T_{s}\right)-\bar{v}_{2}\left(T_{s}\right)\right)^{T}\left(v_{i}\left(T_{s}\right)-\bar{v}_{2}\left(T_{s}\right)\right)+\left(\dot{v}_{i}(t)-\dot{\bar{v}}_{2}(t)\right)^{T}\left(\dot{v}_{i}(t)-\dot{\bar{v}}_{2}(t)\right)\right],
\end{array}, i \in \ell_{2},\right.
\end{aligned}
$$

in which $k_{i}>0$ and $\varepsilon_{i}>0$ are the weights of $c_{i}\left(t_{s}\right)$ and $d_{i}\left(t_{s}\right)$, respectively.

The position's and velocity's weighted coupling configuration matrices of system (1) can represented as

$$
\begin{aligned}
& A=\left[\begin{array}{cc}
A_{11}^{L \times L} & B_{12}^{L \times(N-L)} \\
B_{21}^{(N-L) \times L} & A_{22}^{(N-L) \times(N-L)}
\end{array}\right], \\
& P=\left[\begin{array}{cc}
P_{11}^{L \times L} & Q_{12}^{L \times(N-L)} \\
Q_{21}^{(N-L) \times L} & P_{22}^{(N-L) \times(N-L)}
\end{array}\right],
\end{aligned}
$$

where

$$
A_{11}=\left[\begin{array}{ccc}
a_{11}-\sum_{j=1}^{L} a_{1 j} & \cdots & a_{1 L} \\
\vdots & \ddots & \vdots \\
a_{L 1} & \cdots & a_{L L}-\sum_{j=1}^{L} a_{L j}
\end{array}\right],
$$

$A_{22}=\left[\begin{array}{ccc}a_{(L+1)(L+1)}-\sum_{j=L+1}^{N} a_{(L+1) j} & \cdots & a_{(L+1) N} \\ \vdots & \ddots & \vdots \\ a_{N(L+1)} & \cdots & a_{N N}-\sum_{j=L+1}^{N} a_{N j}\end{array}\right]$,

$P_{11}=\left[\begin{array}{ccc}p_{11}-\sum_{j=1}^{L} p_{1 j} & \cdots & p_{1 L} \\ \vdots & \ddots & \vdots \\ p_{L 1} & \cdots & p_{L L}-\sum_{j=1}^{L} p_{L j}\end{array}\right]$, 
In order to solve the synchronization problem, we briefly give some assumptions, lemmas, and definitions used in this paper.

Assumption 1 (see [39]). The coupling strengths and feedback gains are all bounded, that is,

$$
\begin{aligned}
\left\|c_{i j}\left(t_{s}\right)\right\| & \leq c_{i j},\left\|d_{i j}\left(t_{s}\right)\right\| \leq d_{i j},\left\|c_{i}\left(t_{s}\right)\right\| \leq c_{i},\left\|\alpha_{i j}\left(t_{s}\right)\right\| \\
& \leq \alpha_{i j},\left\|\beta_{i j}\left(t_{s}\right)\right\| \leq \beta_{i j},\left\|d_{i}\left(t_{s}\right)\right\| \leq d_{i},
\end{aligned}
$$

where $\|\cdot\|$ is the Euclidean norm and $c_{i j}, d_{i j}, c_{i}, \alpha_{i j}, \beta_{i j}$, and $d_{i}$ are positive constants. In fact, the coupling strengths and feedback gains are usually bounded.

Assumption 2 (see [39]). $0 \leq \tau(t) \leq \tau$, when $t \geq 0$ and $\tau>0$ are constants.

Unlike some existing works, such as [40], $0 \leq \dot{\tau}(t) \leq 1$ is required; however, this paper does not need to know any information about the derivative of $\tau(t)$.

Assumption $3 \exists \rho_{1}>0, \rho_{2}>0$ such that

$$
\|f(\alpha, \beta)-f(\gamma, \delta)\| \leq \rho_{1}\|\alpha-\gamma\|+\rho_{2}\|\beta-\delta\|, \quad \forall \alpha, \beta, \gamma, \delta \in R^{n},
$$

which can guarantee the boundedness of the nonlinear term for system (1).

Lemma 1 (see [39]). Suppose that $x, y \in R^{N}$ are arbitrary vectors and matrix $Q \in R^{N \times N}$ is positive definite; then, the inequality satisfies

$$
2 x^{T} y \leq x^{T} \mathrm{Q} x+y^{T} \mathrm{Q}^{-1} y .
$$

Lemma 2 (see [39]). If $A=\left(a_{i j}\right) \in R^{N \times N}$ is symmetric irreducible, each eigenvalue of $A-B$ is negative, where $a_{i i}=$ $-\sum_{j=1, j \neq i}^{N} a_{i j}$ and $B=\operatorname{diag}(b, 0, \ldots, 0)$ with $b>0$.

Lemma 3 (see [39]). For an undirected graph G, its corresponding coupling matrix $A$ is irreducible iff $G$ is connected.

Lemma 4 (see [39]). If $\forall x(t) \in R^{n}$ is real differentiable and $W=W^{T}>0$ is a constant matrix, we can have

$$
\begin{gathered}
{\left[\int_{t-\tau(t)}^{t} x(k) \mathrm{d} k\right]^{T} W\left[\int_{t-\tau(t)}^{t} x(k) \mathrm{d} k\right]} \\
\leq \tau \int_{t-\tau(t)}^{t} x^{T}(k) W x(k) \mathrm{d} k, \quad t \geq 0,
\end{gathered}
$$

where $0 \leq \tau(t) \leq \tau$.

\section{Main Results}

For $\alpha>0$ and the sample periodic $\mathbf{T}$, we assume that

$$
t_{i+1}-t_{i}=\alpha \mathbf{T}_{i}, \quad \forall i=0,1,2, \ldots,
$$

where $t_{0}<t_{1}<\cdots$ are the discrete time periods and integer $\mathbf{T}_{i}>0$ is a sampled time with $\mathbf{T}_{i} \leq \mathbf{T}$. Inspired by [36], we design a linear synchronization protocol under the sampling period as

$$
\left\{\begin{array}{l}
\dot{v}_{i}\left(t_{s}+\alpha\right)=\dot{v}_{i}\left(t_{s}\right)-\frac{1}{\mathbf{T}} \dot{v}_{i}\left(t_{s}\right)=\left(1-\frac{1}{\mathbf{T}}\right) \dot{v}_{i}\left(t_{s}\right) \\
\dot{v}_{i}\left(t_{s}+2 \alpha\right)=\dot{v}_{i}\left(t_{s}+\alpha\right)+\left(\dot{v}_{i}\left(t_{s}+\alpha\right)-\dot{v}_{i}\left(t_{s}\right)\right)=\left(1-\frac{2}{\mathbf{T}}\right) \dot{v}_{i}\left(t_{s}\right) \\
\vdots \\
\dot{v}_{i}\left(t_{s+1}-\alpha\right)=\dot{v}_{i}\left(t_{s}+\mathbf{T}_{s} \alpha-\alpha\right)=\left(1-\frac{\mathbf{T}_{s}-1}{\mathbf{T}}\right) \dot{v}_{i}\left(t_{s}\right)
\end{array}\right.
$$

Let $h=0,1, \ldots, \mathbf{T}_{s}-1$; thus, we have

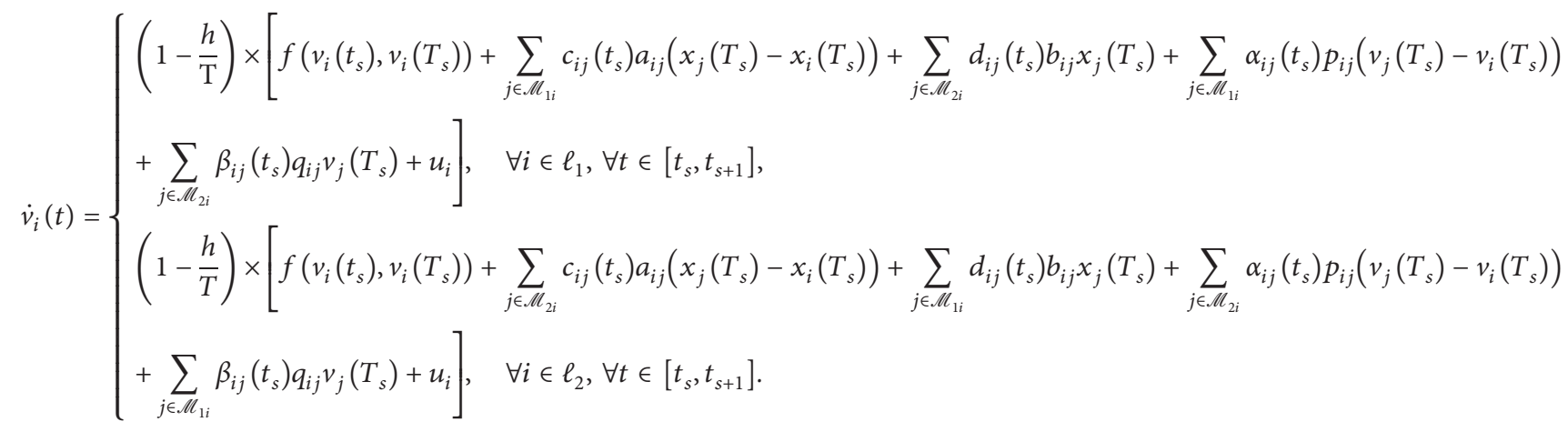


Theorem 1. Consider connected network (1) with control input (2) steered by (3)-(5) under Assumptions 1-3 and Lemmas 1-4; then, each node's position and velocity can asymptotically synchronize.

Proof. Let

$$
\begin{aligned}
& \widetilde{x}_{i}\left(t_{s}\right) \triangleq x_{i}\left(t_{s}\right)-\bar{x}_{1}\left(t_{s}\right), \\
& \widetilde{v}_{i}\left(t_{s}\right) \triangleq v_{i}\left(t_{s}\right)-\bar{v}_{1}\left(t_{s}\right),
\end{aligned}
$$

for $i \in \ell_{1}$, and

$$
\begin{aligned}
& \tilde{x}_{i}\left(t_{s}\right) \triangleq x_{i}\left(t_{s}\right)-\bar{x}_{2}\left(t_{s}\right), \\
& \widetilde{v}_{i}\left(t_{s}\right) \triangleq v_{i}\left(\left(t_{s}\right)-\bar{v}_{2}\left(t_{s}\right),\right.
\end{aligned}
$$

for $i \in \ell_{2}$; then, we obtain

$$
\dot{\tilde{v}}_{i}(t)=\left\{\begin{array}{l}
\left(1-\frac{h}{\mathbf{T}}\right) \times\left[f\left(v_{i}\left(t_{s}\right), v_{i}\left(T_{s}\right)\right)-f\left(\bar{v}_{1}\left(t_{s}\right), \bar{v}_{1}\left(T_{s}\right)\right)+\sum_{j \in \mathscr{M}_{1 i}} c_{i j}\left(t_{s}\right) a_{i j}\left(\widetilde{x}_{j}\left(T_{s}\right)-\widetilde{x}_{i}\left(T_{s}\right)\right)+\sum_{j \in \mathscr{M}_{2 i}} d_{i j}\left(t_{s}\right) b_{i j} \widetilde{x}_{j}\left(T_{s}\right)\right. \\
\left.+\sum_{j \in \mathscr{M}_{1 i}} \alpha_{i j}\left(t_{s}\right) p_{i j}\left(\widetilde{v}_{j}\left(T_{s}\right)-\widetilde{v}_{i}\left(T_{s}\right)\right)+\sum_{j \in \mathscr{M}_{2 i}} \beta_{i j}\left(t_{s}\right) q_{i j} \widetilde{v}_{j}\left(T_{s}\right)+u_{i}\right], \quad \forall i \in \ell_{1}, \forall t \in\left[t_{s}, t_{s+1}\right], \\
\left(1-\frac{h}{\mathbf{T}}\right) \times\left[f\left(v_{i}\left(t_{s}\right), v_{i}\left(T_{s}\right)\right)-f\left(\bar{v}_{2}\left(t_{s}\right), \bar{v}_{2}\left(T_{s}\right)\right)+\sum_{j \in \mathscr{M}_{2 i}} c_{i j}\left(t_{s}\right) a_{i j}\left(\widetilde{x}_{j}\left(T_{s}\right)-\widetilde{x}_{i}\left(T_{s}\right)\right)+\sum_{j \in \mathscr{M}_{1 i}} d_{i j}\left(t_{s}\right) b_{i j} \widetilde{x}_{j}\left(T_{s}\right)\right. \\
\left.+\sum_{j \in \mathscr{M}_{2 i}} \alpha_{i j}\left(t_{s}\right) p_{i j}\left(\widetilde{v}_{j}\left(T_{s}\right)-\widetilde{v}_{i}\left(T_{s}\right)\right)+\sum_{j \in \mathscr{M}_{1 i}} \beta_{i j}\left(t_{s}\right) q_{i j} \widetilde{v}_{j}\left(T_{s}\right)+u_{i}\right], \quad \forall i \in \ell_{2}, \forall t \in\left[t_{s}, t_{s+1}\right] .
\end{array}\right.
$$

Construct a Lyapunov function as

$$
V\left(t_{s}\right)=V_{1}\left(t_{s}\right)+V_{2}\left(t_{s}\right)+V_{3}\left(t_{s}\right) \text {, }
$$

$$
\begin{aligned}
& V_{1}\left(t_{s}\right)=\frac{1}{2} \sum_{i \in \mathscr{M}_{1 i}} \tilde{x}_{i}^{T}\left(t_{s}\right) \tilde{x}_{i}\left(t_{s}\right)+\sum_{i \in \mathscr{M}_{1 i}} \sum_{j \in \mathscr{M}_{1 i}} \frac{\left(c_{i j}\left(t_{s}\right)-2 c_{i j}-p\right)^{2}}{4 k_{i j}}+\sum_{i \in \mathscr{M}_{1 i}} \sum_{j \in \mathscr{M}_{2 i}} \frac{\left(d_{i j}\left(t_{s}\right)-2 d_{i j}-1\right)^{2}}{4 k_{i j}}+\sum_{i \in \mathscr{M}_{1 i}} \frac{\left(c_{i}\left(t_{s}\right)-(3 / 2) c_{i}-p\right)^{2}}{2 k_{i}} \\
& +\frac{1}{2} \sum_{i \in \mathscr{M}_{1 i}} \widetilde{v}_{i}^{T}\left(t_{s}\right) \widetilde{v}_{i}\left(t_{s}\right)+\sum_{i \in \mathscr{M}_{1 i}} \sum_{j \in \mathscr{M}_{1 i}} \frac{\left(p_{i j}\left(t_{s}\right)-2 p_{i j}-p\right)^{2}}{4 \varepsilon_{i j}}+\sum_{i \in \mathscr{M}_{1 i}} \sum_{j \in \mathscr{M}_{2 i}} \frac{\left(q_{i j}\left(t_{s}\right)-2 q_{i j}-1\right)^{2}}{4 \varepsilon_{i j}} \sum_{i \in \mathscr{M}_{1 i}} \frac{\left(d_{i}\left(t_{s}\right)-(3 / 2) d_{i}-p\right)^{2}}{2 \varepsilon_{i}} \\
& V_{2}\left(t_{s}\right)=\frac{1}{2} \sum_{i \in \mathscr{M}_{2 i}} \tilde{x}_{i}^{T}\left(t_{s}\right) \tilde{x}_{i}\left(t_{s}\right)+\sum_{i \in \mathscr{M}_{2 i}} \sum_{j \in \mathscr{M}_{2 i}} \frac{\left(c_{i j}\left(t_{s}\right)-2 c_{i j}-p\right)^{2}}{4 k_{i j}}+\sum_{i \in \mathscr{M}_{2 i}} \sum_{j \in \mathscr{M}_{1 i}} \frac{\left(d_{i j}\left(t_{s}\right)-2 d_{i j}-1\right)^{2}}{4 k_{i j}}+\sum_{i \in \mathscr{M}_{2 i}} \frac{\left(c_{i}\left(t_{s}\right)-(3 / 2) c_{i}-p\right)^{2}}{2 k_{i}} \\
& +\frac{1}{2} \sum_{i \in \mathscr{M}_{2 i}} \widetilde{v}_{i}^{T}\left(t_{s}\right) \widetilde{v}_{i}\left(t_{s}\right)+\sum_{i \in \mathscr{M}_{2 i}} \sum_{j \in \mathscr{M}_{2 i}} \frac{\left(\alpha_{i j}\left(t_{s}\right)-2 \alpha_{i j}-p\right)^{2}}{4 \varepsilon_{i j}}+\sum_{i \in \mathscr{M}_{2 i}} \sum_{j \in \mathscr{M}_{1 i}} \frac{\left(\beta_{i j}\left(t_{s}\right)-2 \beta_{i j}-1\right)^{2}}{4 \varepsilon_{i j}}+\sum_{i \in \mathscr{M}_{2 i}} \frac{\left(d_{i}\left(t_{s}\right)-(3 / 2) d_{i}-p\right)^{2}}{2 \varepsilon_{i}} \\
& V_{3}\left(t_{s}\right)=\tau \sum_{i \in \mathscr{M}_{1 i}}\left[2 \rho_{1}+\rho_{2}+1+\left(\sum_{i \in \mathscr{M}_{1 i}} c_{i j}\left(t_{s}\right) a_{i j}\right)+\left(\sum_{i \in \mathscr{M}_{1 i}} \alpha_{i j}\left(t_{s}\right) p_{i j}\right)+\left(\sum_{i \in \mathscr{M}_{2 i}} d_{i j}\left(t_{s}\right) b_{i j}\right)+\left(\sum_{i \in \mathscr{M}_{2 i}} \beta_{i j}\left(t_{s}\right) q_{i j}\right)\right. \\
& \left.+\left(c_{i}\left(t_{s}\right) h_{i}\right)+\left(d_{i}\left(t_{s}\right) l_{i}\right)\right] \int_{T_{s}}^{t_{s}}\left(k-t_{s}+\tau\right) \dot{\tilde{x}}_{i}^{T}(k) \dot{\tilde{x}}_{i}(k) \mathrm{d} k+\tau \sum_{i \in M_{1 i}} \int_{T_{s}}^{t_{s}}\left(k-t_{s}+\tau\right) \dot{\tilde{x}}_{i}^{T}(k) \dot{\tilde{x}}_{i}(k) \mathrm{d} k \\
& +\tau \sum_{i \in \mathscr{M}_{2 i}}\left[2 \rho_{3}+\rho_{4}+1+\left(\sum_{i \in \mathscr{M}_{2 i}} c_{i j}\left(t_{s}\right) a_{i j}\right)+\left(\sum_{i \in \mathscr{M}_{2 i}} \alpha_{i j}\left(t_{s}\right) p_{i j}\right)+\left(\sum_{i \in \mathscr{M}_{1 i}} d_{i j}\left(t_{s}\right) b_{i j}\right)+\left(\sum_{i \in \mathscr{M}_{1 i}} \beta_{i j}\left(t_{s}\right) q_{i j}\right)\right. \\
& \left.+\left(c_{i}\left(t_{s}\right) h_{i}\right)+\left(d_{i}\left(t_{s}\right) l_{i}\right)\right] \int_{T_{s}}^{t_{s}}\left(k-t_{s}+\tau\right) \dot{\tilde{x}}_{i}^{T}(k) \dot{\tilde{x}}_{i}(k) \mathrm{d} k+\tau \sum_{i \in \mathscr{M}_{2 i}} \int_{T_{s}}^{t_{s}}\left(k-t_{s}+\tau\right) \dot{\tilde{x}}_{i}^{T}(k) \dot{\tilde{x}}_{i}(k) \mathrm{d} k,
\end{aligned}
$$


and $p>0$ is sufficiently large. Next, there are two cases to discuss.
Differentiating $V_{1}\left(t_{s}\right)$, under Assumptions 1-3 and Lemmas 1-4, we can have

Case 1. $A_{11}, A_{22}, P_{11}$, and $P_{22}$ are symmetric.

$$
\begin{aligned}
& \dot{V}_{1}\left(t_{s}\right) \leq \frac{1}{2} \sum_{i \in \mathscr{M}_{1 i}} \tilde{x}_{i}^{T}(t) \widetilde{x}_{i}(t)+\frac{1}{2} \rho_{2} \sum_{i \in \mathscr{M}_{1 i}} \widetilde{v}_{i}^{T}\left(T_{s}\right) \widetilde{v}_{i}\left(T_{s}\right)+\frac{1}{2} \sum_{i \in \mathscr{M}_{1 i}} \xi_{1 i} \widetilde{v}_{i}^{T}(t) \widetilde{v}_{i}(t) \\
& -\frac{p}{2} \sum_{i \in \mathscr{M}_{1 i}} \sum_{j \in \mathscr{M}_{1 i}} a_{i j}\left[\left(\tilde{x}_{i}\left(T_{s}\right)-\tilde{x}_{j}\left(T_{s}\right)\right)^{T}\left(\tilde{x}_{i}\left(T_{s}\right)-\tilde{x}_{j}\left(T_{s}\right)\right)+\left(\dot{\tilde{x}}_{i}(t)-\dot{\tilde{x}}_{j}(t)\right)^{T}\left(\dot{\tilde{x}}_{i}(t)-\dot{\tilde{x}}_{j}(t)\right)\right] \\
& -\frac{p}{2} \sum_{i \in \mathscr{M}_{1 i}} \sum_{j \in \mathscr{M}_{1 i}} p_{i j}\left[\left(\widetilde{v}_{i}\left(T_{s}\right)-\widetilde{v}_{j}\left(T_{s}\right)\right)^{T}\left(\widetilde{v}_{i}\left(T_{s}\right)-\widetilde{v}_{j}\left(T_{s}\right)\right)+\left(\dot{\tilde{v}}_{i}(t)-\dot{\tilde{v}}_{j}(t)\right)^{T}\left(\dot{\tilde{v}}_{i}(t)-\dot{\tilde{v}}_{j}(t)\right)\right] \\
& -\frac{1}{2} \sum_{i \in \mathscr{M}_{1 i}} \sum_{j \in \mathscr{M}_{2 i}} b_{i j} \tilde{x}_{j}^{T}\left(T_{s}\right) \tilde{x}_{j}\left(T_{s}\right)-\frac{1}{2} \sum_{i \in \mathscr{M}_{1 i}} \sum_{j \in \mathscr{M}_{2 i}} q_{i j} \widetilde{v}_{j}^{T}\left(T_{s}\right) \widetilde{v}_{j}\left(T_{s}\right) \\
& -\frac{p}{2} \sum_{i \in \mathscr{M}_{1 i}} h_{i}\left[\tilde{x}_{i}^{T}\left(T_{s}\right) \tilde{x}_{i}\left(T_{s}\right)+\dot{\tilde{x}}_{i}^{T}(t) \dot{\tilde{x}}_{i}(t)\right]-\frac{p}{2} \sum_{i \in \mathscr{M}_{1 i}} l_{i}\left[\widetilde{v}_{i}^{T}\left(T_{s}\right) \widetilde{v}_{i}\left(T_{s}\right)+\dot{\tilde{v}}_{i}^{T}(t) \dot{\tilde{v}}_{i}(t)\right],
\end{aligned}
$$

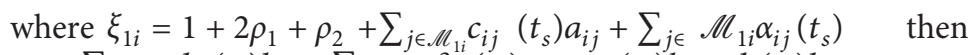
$p_{i j}+\sum_{j \in M_{2 i}} d_{i j}\left(t_{s}\right) b_{i j}+\sum_{j \in \mathscr{M}_{2 i}} \beta_{i j}\left(t_{s}\right) q_{i j}+c_{i}\left(t_{s}\right) h_{i}+d_{i}\left(t_{s}\right) l_{i}$.

Using Leibniz-Newton formula,

$$
x\left(t_{s}\right)-x\left(T_{s}\right)=\int_{T_{s}}^{t_{s}} \dot{x}(k) \mathrm{d} k
$$

$$
\left(x\left(t_{s}\right)\right)^{T}=\left(x\left(T_{s}\right)\right)^{T}+\left(\int_{T_{s}}^{t_{s}} \dot{x}(k) \mathrm{d} k\right)^{T},
$$

and then

$$
\begin{aligned}
\left(\tilde{x}\left(t_{s}\right)\right)^{T}\left(\tilde{x}\left(t_{s}\right)\right) & =\left[\left(\tilde{x}\left(T_{s}\right)\right)^{T}+\left(\int_{T_{s}}^{t_{s}} \dot{\tilde{x}}(k) \mathrm{d} k\right)^{T}\right]\left[\left(\tilde{x}\left(T_{s}\right)\right)+\left(\int_{T_{s}}^{t_{s}} \dot{\tilde{x}}(k) \mathrm{d} k\right)\right] \\
& \leq 2\left(\widetilde{x}\left(T_{s}\right)\right)^{T}\left(\tilde{x}\left(T_{s}\right)\right)+2\left(\int_{T_{s}}^{t_{s}} \dot{\tilde{x}}(k) \mathrm{d} k\right)^{T}\left(\int_{T_{s}}^{t_{s}} \dot{\tilde{x}}(k) \mathrm{d} k\right) \\
& \leq 2\left(\tilde{x}\left(T_{s}\right)\right)^{T}\left(\tilde{x}\left(T_{s}\right)\right)+2 \tau \int_{V_{s}}^{t_{s}} \dot{\tilde{x}}_{i}^{T}(k) \dot{\tilde{x}}_{i}(k) \mathrm{d} k .
\end{aligned}
$$

Similarly,

$\left(\widetilde{v}\left(t_{s}\right)\right)^{T}\left(\widetilde{v}\left(t_{s}\right)\right) \leq 2\left(\widetilde{v}\left(T_{s}\right)\right)^{T}\left(\widetilde{v}\left(T_{s}\right)\right)+2 \tau \int_{T_{s}}^{t_{s}} \dot{\widetilde{v}}_{i}^{T}(k) \dot{\tilde{v}}_{i}(k) \mathrm{d} k$. 


$$
\begin{aligned}
\dot{V}_{1}\left(t_{s}\right) \leq & \sum_{i \in \mathscr{M}_{1 i}} \tilde{x}_{i}^{T}\left(T_{s}\right) \widetilde{x}_{i}\left(T_{s}\right)+\tau \sum_{i \in \mathscr{M}_{1 i}} \int_{T_{s}}^{t_{s}} \dot{\tilde{x}}_{i}^{T}(k) \dot{\tilde{x}}_{i}(k) \mathrm{d} k-\frac{1}{2} \sum_{i \in \mathscr{M}_{1 i}} \sum_{j \in \mathscr{M}_{2 i}} b_{i j} \widetilde{x}_{j}^{T}\left(T_{s}\right) \widetilde{x}_{j}^{T}\left(T_{s}\right) \\
& +\sum_{i \in \mathscr{M}_{1 i}}\left(\xi_{1 i}+\frac{1}{2} \rho_{2}\right) \widetilde{v}_{i}^{T}\left(T_{s}\right) \widetilde{v}_{i}\left(T_{s}\right)+\tau \sum_{i \in \mathscr{M}_{1 i}} \xi_{1 i} \int_{T_{s}}^{t_{s}} \dot{\widetilde{v}}_{i}^{T}(k) \dot{\vec{v}}_{i}(k) \mathrm{d} k-\frac{1}{2} \sum_{i \in \mathscr{M}_{1 i}} \sum_{j \in \mathscr{M}_{2 i}} q_{i j} \widetilde{v}_{j}^{T}\left(T_{s}\right) \widetilde{v}_{j}\left(T_{s}\right) \\
& +\sum_{i \in \mathscr{M}_{1 i}} p \lambda_{1}\left\|\tilde{x}_{i}\left(T_{s}\right)\right\|^{2}+\sum_{i \in \mathscr{M}_{1 i}} p \lambda_{1}\left\|\dot{\tilde{x}}_{i}\left(t_{s}\right)\right\|^{2}+\sum_{i \in \mathscr{M}_{1 i}} p \lambda_{3}\left\|\widetilde{v}_{i}\left(T_{s}\right)\right\|^{2}+\sum_{i \in \mathscr{M}_{1 i}} p \lambda_{3}\left\|\dot{\tilde{v}}_{i}\left(t_{s}\right)\right\|^{2},
\end{aligned}
$$

where $\quad \xi_{1 i}=1+2 \rho_{1}+\rho_{2}+\sum_{j \in M_{1 i}} c_{i j}\left(t_{s}\right) a_{i j}+\sum_{j \in \mathscr{M}_{1 i}} \alpha_{i j}\left(t_{s}\right)$

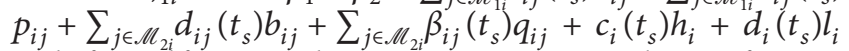
and $\lambda_{1}$ and $\lambda_{3}$ are the minimum eigenvalues of $A_{11}-$ $H_{1}$ and $P_{11}-L_{1}$, respectively.
Similarly, differentiating $V_{2}\left(t_{s}\right)$ and $V_{3}\left(t_{s}\right)$, we can obtain

$$
\begin{aligned}
\dot{V}_{2}\left(t_{s}\right) \leq & \sum_{i \in \mathscr{M}_{2 i}} \tilde{x}_{i}^{T}\left(T_{s}\right) \tilde{x}_{i}\left(T_{s}\right)+\tau \sum_{i \in \mathscr{M}_{2 i}} \int_{T_{s}}^{t_{s}} \dot{\tilde{x}}_{i}^{T}(k) \dot{\tilde{x}}_{i}(k) \mathrm{d} k-\frac{1}{2} \sum_{i \in \mathscr{M}_{2 i}} \sum_{j \in \mathscr{M}_{1 i}} b_{i j} \widetilde{x}_{j}^{T}\left(T_{s}\right) \tilde{x}_{j}\left(T_{s}\right) \\
& +\sum_{i \in \mathscr{M}_{2 i}}\left(\xi_{2 i}+\frac{1}{2} \rho_{4}\right) \widetilde{v}_{i}^{T}\left(T_{s}\right) \widetilde{v}_{i}\left(T_{s}\right)+\tau \sum_{i \in \mathscr{M}_{2 i}} \xi_{2 i} \int_{T_{s}}^{t_{s}} \dot{\widetilde{v}}_{i}^{T}(k) \dot{\tilde{v}}_{i}(k) \mathrm{d} k-\frac{1}{2} \sum_{i \in \mathscr{M}_{2 i}} \sum_{j \in \mathscr{M}_{1 i}} q_{i j} \widetilde{v}_{j}^{T}\left(T_{s}\right) \widetilde{v}_{j}\left(T_{s}\right) \\
& +\sum_{i \in \mathscr{M}_{2 i}} p \lambda_{2}\left\|\tilde{x}_{i}\left(T_{s}\right)\right\|^{2}+\sum_{i \in \mathscr{M}_{2 i}} p \lambda_{2}\left\|\dot{\tilde{x}}_{i}\left(t_{s}\right)\right\|^{2}+\sum_{i \in \mathscr{M}_{2 i}} p \lambda_{4}\left\|\widetilde{v}_{i}\left(T_{s}\right)\right\|^{2}+\sum_{i \in \mathscr{M}_{2 i}} p \lambda_{4}\left\|\tilde{\tilde{v}}_{i}\left(t_{s}\right)\right\|^{2},
\end{aligned}
$$

where $\quad \xi_{2 i}=1+2 \rho_{3}+\rho_{4}+\sum_{j \in \mathscr{M}_{2 i}} c_{i j}\left(t_{s}\right) a_{i j}+\sum_{j \in \mathscr{M}_{2 i}} \alpha_{i j}\left(t_{s}\right) \quad$ and $\lambda_{2}$ and $\lambda_{4}$ are the minimum eigenvalues of $A_{22}-H_{2}$ $p_{i j}+\sum_{j \in \mathscr{M}_{1 i}} d_{i j}\left(t_{s}\right) b_{i j}+\sum_{j \in \mathscr{M}_{1 i}} \beta_{i j}\left(t_{s}\right) q_{i j}+c_{i}\left(t_{s}\right) h_{i}+d_{i}\left(t_{s}\right) l_{i} \quad$ and $P_{22}-L_{2}$, respectively:

$$
\begin{aligned}
\dot{V}_{3}\left(t_{s}\right)= & \tau^{2} \sum_{i \in \mathscr{M}_{1 i}} \dot{\tilde{x}}_{i}^{T}\left(t_{s}\right) \dot{\tilde{x}}_{i}\left(t_{s}\right)-\tau \sum_{i \in \mathscr{M}_{1 i}} \int_{T_{s}}^{t_{s}} \dot{\tilde{x}}_{i}^{T}(k) \dot{\tilde{x}}_{i}(k) \mathrm{d} k+\tau^{2} \sum_{i \in \mathscr{M}_{1 i}} \xi_{1 i} \dot{\tilde{v}}_{i}^{T}\left(t_{s}\right) \dot{\tilde{v}}_{i}\left(t_{s}\right)-\tau \sum_{i \in \mathscr{M}_{1 i}} \xi_{1 i} \int_{T_{s}}^{t_{s}} \dot{\tilde{v}}_{i}^{T}(k) \dot{\tilde{v}}_{i}(k) \mathrm{d} k \\
& +\tau^{2} \sum_{i \in \mathscr{M}_{2 i}} \dot{\tilde{x}}_{i}^{T}\left(t_{s}\right) \dot{\tilde{x}}_{i}\left(t_{s}\right)-\tau \sum_{i \in \mathscr{M}_{2 i}} \int_{T_{s}}^{t_{s}} \dot{\tilde{x}}_{i}^{T}(k) \dot{\tilde{x}}_{i}(k) \mathrm{d} k+\tau^{2} \sum_{i \in \mathscr{M}_{2 i}} \xi_{2 i} \dot{\tilde{v}}_{i}^{T}\left(t_{s}\right) \dot{\tilde{v}}_{i}\left(t_{s}\right)-\tau \sum_{i \in \mathscr{M}_{2 i}} \xi_{2 i} \int_{T_{s}}^{t_{s}} \dot{\tilde{v}}_{i}^{T}(k) \dot{\bar{v}}_{i}(k) \mathrm{d} k .
\end{aligned}
$$

Combining $\dot{V}_{1}\left(t_{s}\right), \dot{V}_{2}\left(t_{s}\right)$, and $\dot{V}_{3}\left(t_{s}\right)$, we obtain

$$
\begin{aligned}
\dot{V}\left(t_{s}\right) \leq & \sum_{i \in \mathscr{M}_{1 i}}\left(1+p \lambda_{1}\right)\left\|\tilde{x}_{i}\left(T_{s}\right)\right\|^{2}+\sum_{i \in \mathscr{M}_{1 i}}\left(\tau^{2}+p \lambda_{1}\right)\left\|\dot{\tilde{x}}_{i}\left(t_{s}\right)\right\|^{2}+\sum_{i \in \mathscr{M}_{2 i}}\left(1+p \lambda_{2}\right)\left\|\widetilde{x}_{i}\left(T_{s}\right)\right\|^{2}+\sum_{i \in \mathscr{M}_{2 i}}\left(\tau^{2}+p \lambda_{2}\right)\left\|\dot{\tilde{x}}_{i}\left(t_{s}\right)\right\|^{2} \\
& +\sum_{i \in \mathscr{M}_{1 i}}\left(\xi_{1 i}+\frac{1}{2} \rho_{2}-\frac{1}{2} \sum_{j \in \mathscr{M}_{2 i}} b_{j i}-\frac{1}{2} \sum_{j \in \mathscr{M}_{2 i}} q_{j i}+p \lambda_{3}\right)\left\|\widetilde{v}_{i}\left(T_{s}\right)\right\|^{2}+\sum_{i \in \mathscr{M}_{1 i}}\left(\tau^{2} \xi_{1 i}+p \lambda_{3}\right)\left\|\dot{\tilde{v}}_{i}\left(t_{s}\right)\right\|^{2} \\
& +\sum_{i \in \mathscr{M}_{2 i}}\left(\xi_{2 i}+\frac{1}{2} \rho_{4}-\frac{1}{2} \sum_{j \in \mathscr{M}_{1 i}} b_{j i}-\frac{1}{2} \sum_{j \in \mathscr{M}_{1 i}} q_{j i}+p \lambda_{4}\right)\left\|\tilde{v}_{i}\left(T_{s}\right)\right\|^{2}+\sum_{i \in \mathscr{M}_{2 i}}\left(\tau^{2} \xi_{2 i}+p \lambda_{4}\right)\left\|\dot{\tilde{v}}_{i}\left(t_{s}\right)\right\|^{2},
\end{aligned}
$$


where $\lambda_{1}, \lambda_{2}, \lambda_{3}$, and $\lambda_{4}$ are the minimum eigenvalues of $A_{11}-H_{1}, A_{22}-H_{2}, P_{11}-L_{1}$, and $P_{22}-L_{2}$, respectively; with $H_{1}=\operatorname{diag}\left\{h_{i}\right\}$ and $L_{1}=\operatorname{diag}\left\{l_{i}\right\}$ for $\forall i \in \ell_{l}$, and $H_{2}=$ $\operatorname{diag}\left\{h_{i}\right\}$ and $L_{2}=\operatorname{diag}\left\{l_{i}\right\}$ for $\forall i \in \ell_{2}$. Furthermore, since $A_{11}, A_{22}, P_{11}$, and $P_{22}$ are both symmetric and diagonal matrices $H_{1}, H_{2}, L_{1}$, and $L_{2}$ have at least one element being $1, \lambda_{i}<0, i=1,2,3,4$ based on Lemma 2 . Therefore, $\dot{V}\left(t_{s}\right)<0$, if $p>0$ is sufficiently large.
Case 2. $A_{11}, A_{22}, P_{11}$, and $P_{22}$ are asymmetric.

It is known that $\left(A_{11}+A_{11}^{T}\right) / 2,\left(A_{22}+A_{22}^{T}\right) / 2$, $\left(P_{11}+P_{11}^{T}\right) / 2$, and $\left(P_{22}+P_{22}^{T}\right) / 2$ are symmetric, even if $A_{11}, A_{22}, P_{11}$, and $P_{22}$ are asymmetric. Therefore, all eigenvalues of $\left(A_{11}+A_{11}^{T} / 2\right)-H_{1},\left(A_{22}+A_{22}^{T} / 2\right)-H_{2},\left(P_{11}+\right.$ $\left.P_{11}^{T} / 2\right)-L_{1}$, and $\left(P_{22}+P_{22}^{T} / 2\right)-L_{2}$ are negative from Lemma 2. Similarly, we can have

$$
\begin{aligned}
\dot{V}_{1}\left(t_{s}\right) \leq & \sum_{i \in \mathscr{M}_{1 i}} \tilde{x}_{i}^{T}\left(T_{s}\right) \tilde{x}_{i}\left(T_{s}\right)+\tau \sum_{i \in \mathscr{M}_{1 i}} \int_{T_{s}}^{t_{s}} \dot{\tilde{x}}_{i}^{T}(k) \dot{\tilde{x}}_{i}(k) \mathrm{d} k-\frac{1}{2} \sum_{i \in \mathscr{M}_{1 i}} \sum_{\epsilon \in \mathscr{M}_{2 i}} b_{i j} \widetilde{x}_{j}^{T}\left(T_{s}\right) \tilde{x}_{j}\left(T_{s}\right) \\
& +\sum_{i \in \mathscr{M}_{1 i}}\left(\xi_{1 i}+\frac{1}{2} \rho_{2}\right) \widetilde{v}_{i}^{T}\left(T_{s}\right) \widetilde{v}_{i}\left(T_{s}\right)+\tau \sum_{i \in \mathscr{M}_{1 i}} \xi_{1 i} \int_{T_{s}}^{t_{s}} \dot{\widetilde{v}}_{i}^{T}(k) \dot{\vec{v}}_{i}(k) \mathrm{d} k-\frac{1}{2} \sum_{i \in \mathscr{M}_{1 i}} \sum_{j \in \mathscr{M}_{2 i}} q_{i j} \widetilde{v}_{j}^{T}\left(T_{s}\right) \widetilde{v}_{j}\left(T_{s}\right) \\
& +\sum_{i \in \mathscr{M}_{1 i}} p \lambda_{1}\left\|\tilde{x}_{i}\left(T_{s}\right)\right\|^{2}+\sum_{i \in \mathscr{M}_{1 i}} p \lambda_{1}\left\|\dot{\tilde{x}}_{i}\left(t_{s}\right)\right\|^{2}+\sum_{i \in \mathscr{M}_{1 i}} p \lambda_{3}\left\|\tilde{v}_{i}\left(T_{s}\right)\right\|^{2}+\sum_{i \in \mathscr{M}_{1 i}} p \lambda_{3}\left\|\dot{\tilde{v}}_{i}\left(t_{s}\right)\right\|^{2},
\end{aligned}
$$

where $\left.\quad \xi_{2 i}=1+2 \rho_{3}+\rho_{4}+\sum_{j \in \mathscr{M}_{2} c_{i j}} c_{s}\right) a_{i j}+\sum_{j \in \mathscr{M}_{2}} \alpha_{i j}\left(t_{s}\right) \quad$ and $\lambda_{2}$ and $\lambda_{4}$ are the minimum eigenvalue of $\left(A_{22}+\right.$ $\left.p_{i j}+\sum_{j \in \mathscr{M}_{1 i}} d_{i j}\left(t_{s}\right) b_{i j}+\sum_{j \in \mathscr{M}_{1 i}} \beta_{i j}\left(t_{s}\right) q_{i j}+c_{i}\left(t_{s}\right) h_{i}+d_{i}\left(t_{s}\right) l_{i}, \quad A_{22}^{T} / 2\right)-H_{2}$ and $\left(P_{22}+P_{22}^{T} / 2\right)-L_{2}$, respectively.

$$
\begin{aligned}
\dot{V}_{2}\left(t_{s}\right) \leq & \sum_{i \in \mathscr{M}_{2 i}} \tilde{x}_{i}^{T}\left(T_{s}\right) \tilde{x}_{i}\left(T_{s}\right)+\tau \sum_{i \in \mathscr{M}_{2 i}} \int_{T_{s}}^{t_{s}} \dot{\tilde{x}}_{i}^{T}(k) \dot{\tilde{x}}_{i}(k) \mathrm{d} k-\frac{1}{2} \sum_{i \in \mathscr{M}_{2 i}} \sum_{j \in \mathscr{M}_{1 i}} b_{i j} \widetilde{x}_{j}^{T}\left(T_{s}\right) \tilde{x}_{j}\left(T_{s}\right) \\
& +\sum_{i \in \mathscr{M}_{2 i}}\left(\xi_{2 i}+\frac{1}{2} \rho_{4}\right) \tilde{v}_{i}^{T}\left(T_{s}\right) \widetilde{v}_{i}\left(T_{s}\right)+\tau \sum_{i \in \mathscr{M}_{2 i}} \xi_{2 i} \int_{T_{s}}^{t_{s}} \dot{\tilde{v}}_{i}^{T}(k) \dot{\tilde{v}}_{i}(k) \mathrm{d} k-\frac{1}{2} \sum_{i \in \mathscr{M}_{2 i}} \sum_{\epsilon \in \mathscr{M}_{1 i}} q_{i j} \widetilde{v}_{j}^{T}\left(T_{s}\right) \widetilde{v}_{j}\left(T_{s}\right) \\
& +\sum_{i \in \mathscr{M}_{2 i}} p \lambda_{2}\left\|\tilde{x}_{i}\left(T_{s}\right)\right\|^{2}+\sum_{i \in \mathscr{M}_{2 i}} p \lambda_{2}\left\|\dot{\tilde{x}}_{i}\left(t_{s}\right)\right\|^{2}+\sum_{i \in \mathscr{M}_{2 i}} p \lambda_{4}\left\|\tilde{v}_{i}\left(T_{s}\right)\right\|^{2}+\sum_{i \in \mathscr{M}_{2 i}} p \lambda_{4}\left\|\dot{\tilde{v}}_{i}\left(t_{s}\right)\right\|^{2},
\end{aligned}
$$

where $\quad \xi_{1 i}=1+2 \rho_{1}+\rho_{2}+\sum_{j \in \mathscr{M}_{1 i}} \quad c_{i j}\left(t_{s}\right) a_{i j}+\sum_{j \in \mathscr{M}_{1 i}} \alpha_{i j}$ $\left(t_{s}\right) p_{i j}+\sum_{j \in \mathscr{M}_{2 i}} d_{i j}\left(t_{s}\right) b_{i j}+\sum_{j \in \mathscr{M}_{2 i}} \beta_{i j}\left(t_{s}\right) \quad q_{i j}+c_{i}\left(t_{s}\right) h_{i}+d_{i}$ $\left(t_{s}\right) l_{i}$. and $\lambda_{1}$ and $\lambda_{3}$ are the minimum eigenvalue of $\left(A_{11}+A_{11}^{T} / 2\right)-H_{1},\left(P_{11}+P_{11}^{T} / 2\right)-L_{1}$, respectively:

Combining $\dot{V}_{1}\left(t_{s}\right), \dot{V}_{2}\left(t_{s}\right)$, and $\dot{V}_{3}\left(t_{s}\right)$, we obtain

$$
\begin{aligned}
\dot{V}\left(t_{s}\right) \leq & \sum_{i \in \mathscr{M}_{1 i}}\left(1+p \lambda_{1}\right)\left\|\tilde{x}_{i}\left(T_{s}\right)\right\|^{2}+\sum_{i \in \mathscr{M}_{1 i}}\left(\tau^{2}+p \lambda_{1}\right)\left\|\dot{\tilde{x}}_{i}\left(t_{s}\right)\right\|^{2}+\sum_{i \in \mathscr{M}_{2 i}}\left(1+p \lambda_{2}\right)\left\|\tilde{x}_{i}\left(T_{s}\right)\right\|^{2}+\sum_{i \in \mathscr{M}_{2 i}}\left(\tau^{2}+p \lambda_{2}\right)\left\|\dot{\tilde{x}}_{i}\left(t_{s}\right)\right\|^{2} \\
& +\sum_{i \in \mathscr{M}_{1 i}}\left(\xi_{1 i}+\frac{1}{2} \rho_{2}-\frac{1}{2} \sum_{j \in \mathscr{M}_{2 i}} b_{j i}-\frac{1}{2} \sum_{j \in \mathscr{M}_{2 i}} q_{j i}+p \lambda_{3}\right)\left\|\tilde{v}_{i}\left(T_{s}\right)\right\|^{2}+\sum_{i \in \mathscr{M}_{1 i}}\left(\tau^{2} \xi_{1 i}+p \lambda_{3}\right)\left\|\dot{\tilde{v}}_{i}\left(t_{s}\right)\right\|^{2} \\
& +\sum_{i \in \cdot \mathscr{M}_{2 i}}\left(\xi_{2 i}+\frac{1}{2} \rho_{4}-\frac{1}{2} \sum_{j \in \mathscr{M}_{1 i}} b_{j i}-\frac{1}{2} \sum_{j \in \mathscr{M}_{1 i}} q_{j i}+p \lambda_{4}\right)\left\|\tilde{v}_{i}\left(T_{s}\right)\right\|^{2}+\sum_{i \in \mathscr{M}_{2 i}}\left(\tau^{2} \xi_{2 i}+p \lambda_{4}\right)\left\|\dot{\tilde{v}}_{i}\left(t_{s}\right)\right\|^{2},
\end{aligned}
$$


where $\lambda_{1}, \lambda_{2}, \lambda_{3}$, and $\lambda_{4}$ are the minimum eigenvalue of $\left(A_{11}+A_{11}^{T} / 2\right)-H_{1},\left(A_{22}+A_{22}^{T} / 2\right)-H_{2},\left(P_{11}+P_{11}^{T} / 2\right)-L_{1}$, and $\left(P_{22}+P_{22}^{T} / 2\right)-L_{2}$, respectively. Even though matrix $A_{11}, A_{22}, P_{11}$, and $P_{22}$ are asymmetric, matrix $\left(A_{11}+A_{11}^{T}\right) / 2$, $\left(A_{22}+A_{22}^{T}\right) / 2,\left(P_{11}+P_{11}^{T}\right) / 2$, and $\left(P_{22}+P_{22}^{T}\right) / 2$ are symmetric; thus, from Lemma 2, we can obtain all eigenvalues of $\left(A_{11}+A_{11}^{T} / 2\right)-H_{1},\left(A_{22}+A_{22}^{T} / 2\right)-H_{2},\left(P_{11}+P_{11}^{T} / 2\right)-L_{1}$, and $\left(P_{22}+P_{22}^{T} / 2\right)-L_{2}$ are negative. So, $\lambda_{i}<0, \forall i \in 1,2,3,4$ and $\dot{V}\left(t_{s}\right)<0$, if $p>0$ is sufficiently large.

Therefore, all the agents of sampled-data based network (1) with time-varying delays can achieve the given synchronous states asymptotically.

Remark 1. When the topology structure is connected regardless of the coupled weighted matrices, the sampled-databased network (1) with time-varying delays can be asymptotically group synchronized by controller (2).

\section{Simulations}

A complex dynamical system with $N=6$ and $L=3$. Let the

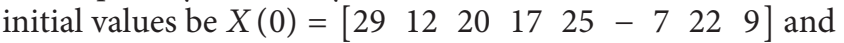
$c_{i j}(0)=d_{i j}(0)=\alpha_{i j}(0)=\beta_{i j}(0)=c_{i}(0)=d_{i}(0)=0.01$.

Let $A_{11}, A_{22}, P_{11}$, and $P_{22}$ be symmetric as

$$
\begin{aligned}
& A_{11}=\left[\begin{array}{ccc}
-2 & 1 & 0 \\
1 & -3 & 1 \\
0 & 1 & -1
\end{array}\right] * 0.1, \\
& A_{22}=\left[\begin{array}{ccc}
-3 & 2 & 1 \\
2 & -4 & 1 \\
1 & 1 & -3
\end{array}\right] * 0.05, \\
& P_{11}=\left[\begin{array}{lll}
0 & 2 & 0 \\
2 & 0 & 1 \\
0 & 1 & 0
\end{array}\right] * 0.01, \\
& P_{22}=\left[\begin{array}{lll}
0 & 2 & 1 \\
2 & 0 & 2 \\
1 & 2 & 0
\end{array}\right] * 0.005,
\end{aligned}
$$

and $A_{11}, A_{22}, P_{11}$, and $P_{22}$ be asymmetric as

$$
\begin{aligned}
& A_{11}=\left[\begin{array}{ccc}
-3 & 0 & 3 \\
2 & -3 & 1 \\
0 & 1 & -1
\end{array}\right] * 0.1, \\
& A_{22}=\left[\begin{array}{ccc}
-2 & 2 & 0 \\
3 & -6 & 3 \\
1 & 2 & -3
\end{array}\right] * 0.05, \\
& P_{11}=\left[\begin{array}{lll}
0 & 3 & 0 \\
2 & 0 & 1 \\
0 & 1 & 0
\end{array}\right] * 0.01, \\
& P_{22}=\left[\begin{array}{lll}
0 & 2 & 0 \\
3 & 0 & 3 \\
0 & 2 & 0
\end{array}\right] * 0.005,
\end{aligned}
$$

respectively.

Take

$$
B_{12}=\left[\begin{array}{lll}
0 & 1 & 0 \\
2 & 0 & 0 \\
0 & 0 & 0.5
\end{array}\right] * 0.1 \text {, }
$$$$
B_{21}=\left[\begin{array}{lll}
1 & 2 & 0 \\
0 & 0 & 0 \\
0 & 0 & 0
\end{array}\right] * 0.1,
$$

$$
\begin{aligned}
& H_{1}=\operatorname{diag}\left\{\begin{array}{lll}
0.1 & 0 & 0
\end{array}\right\}, \\
& H_{2}=\operatorname{diag}\left\{\begin{array}{lll}
0 & 0.1 & 0
\end{array}\right\} .
\end{aligned}
$$

Figure 1 presents that the effects of adaptive strategies for the synchronization of complex networks with nonlinear dynamical. Figure 1(a) shows the position and velocity of all nodes without adaptive strategies, and Figure 1(b) shows the position and velocity of all nodes with adaptive laws, respectively, in which the subgroups' coupling matrices are symmetric. It is obvious to see the fact that all the nodes with adaptive laws can achieve their given synchronous states asymptotically, while all the nodes without adaptive laws cannot converge. Figures 2 and 3 show the simulations of network (1) with $\tau=0.1$, in which the subnetworks' coupling matrices are symmetric or asymmetric descried as Figures 2 and 3, respectively. Figures 2(a) and 2(b) present that the position and velocity of all nodes of network (1) with $\tau=0.1$, where subgroups' coupling matrices are symmetric, and the coupling strengths $c_{i j}, d_{i j} \alpha_{i j}$, and $\beta_{i j}$ and the feedback gains $c_{i}$ and $d_{i}$ are presented in Figures $2(\mathrm{c})-2(\mathrm{~h})$, respectively. Similarly, Figures 3(a) and 3(b) present that the position and velocity of all nodes of network (1) with $\tau=0.1$, where the subgroups' coupling matrices are asymmetric, the coupling strengths $c_{i j}, d_{i j} \alpha_{i j}$, and $\beta_{i j}$ and the feedback gains $c_{i}$ and $d_{i}$ presented as Figures $3(\mathrm{c})-3(\mathrm{~h})$, respectively. From Figures 2 and 3, we can find that all nodes of network (1) can achieve synchronization and the coupling strengths and the feedback gains also converge to be consistent. However, compared with Figure 3, the system in Figure 2 can achieve synchronization faster than that in Figure 3. Figure 4 is the simulation of network (1) with adaptive laws, in which the subgroups' coupling matrices are symmetric, where Figures 4(a) and 4(b) are the positions and velocities of all nodes of network (1) with $\tau=0.1$ and $\tau=1$, respectively. We can know that, with the time delay $\tau$ increasing, the system cannot achieve synchronization. 

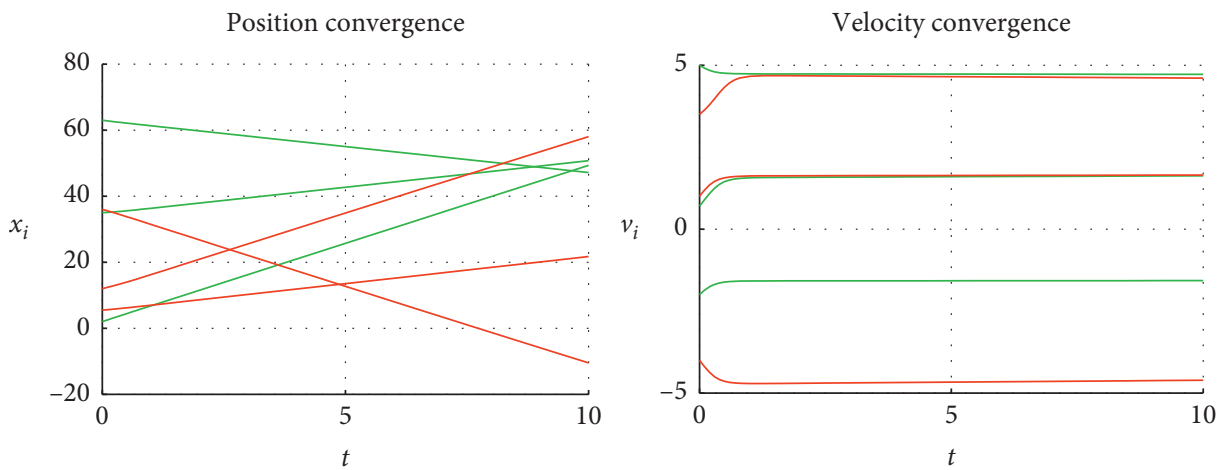

(a)
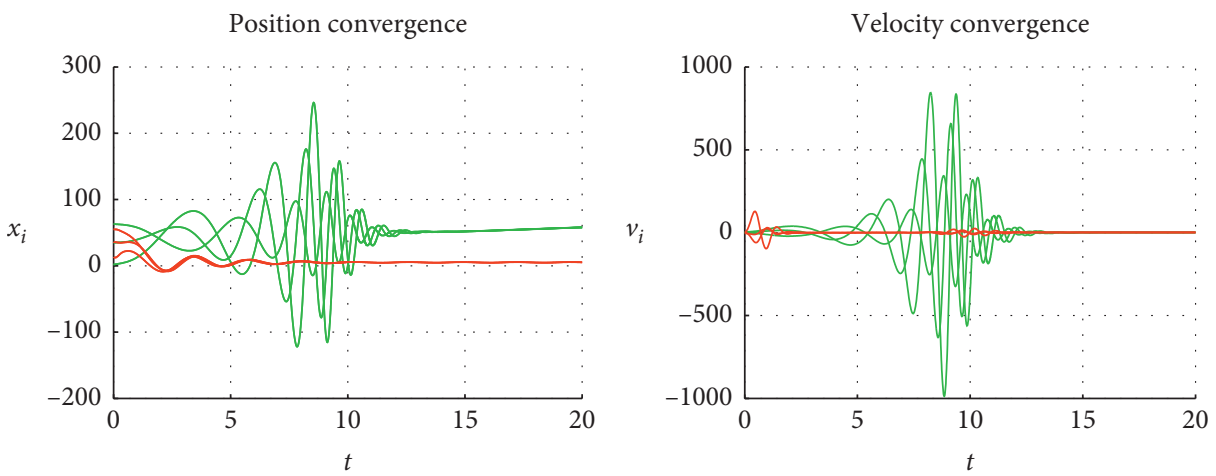

(b)

Figure 1: (a) Without adaptive strategies. (b) With adaptive strategies.

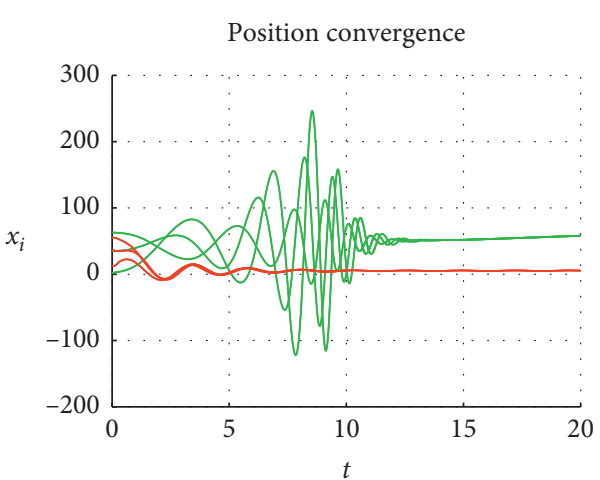

(a)

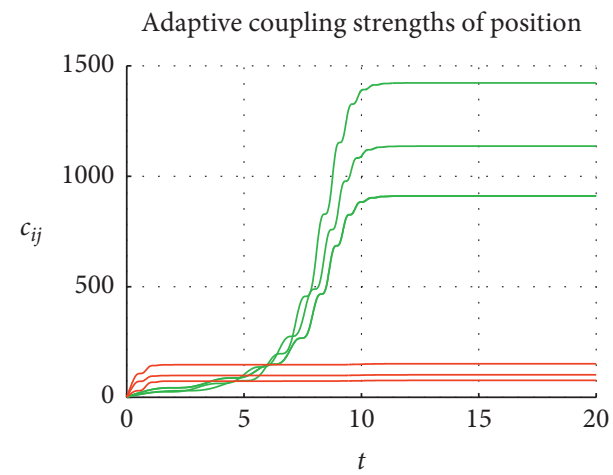

(c)

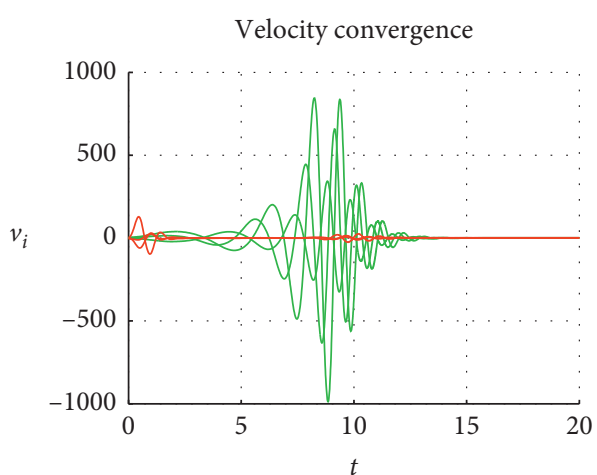

(b)

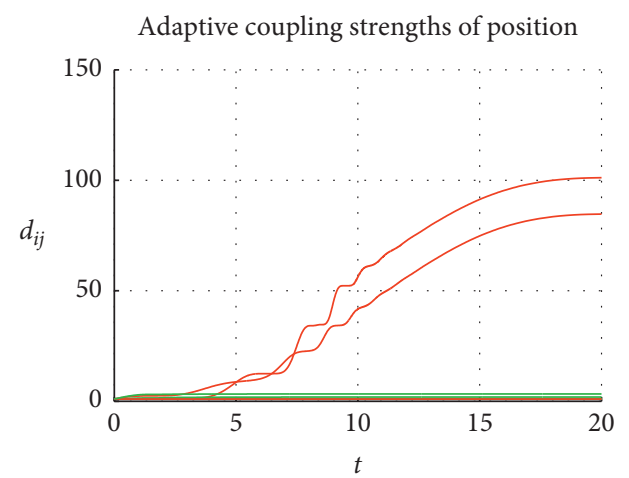

(d)

Figure 2: Continued. 


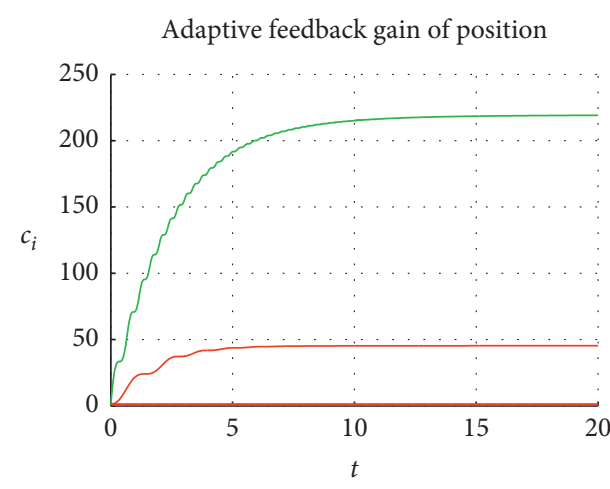

(e)

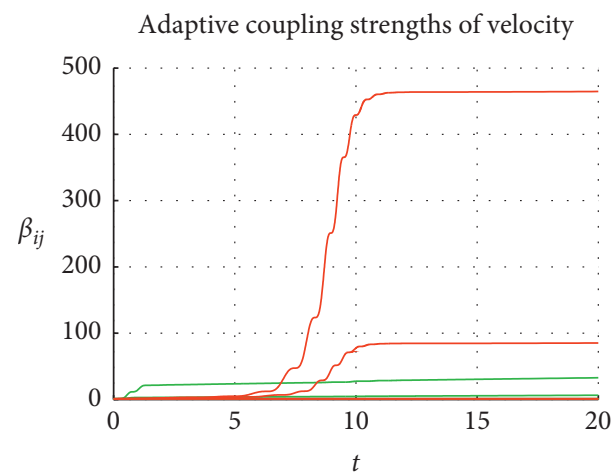

(g)

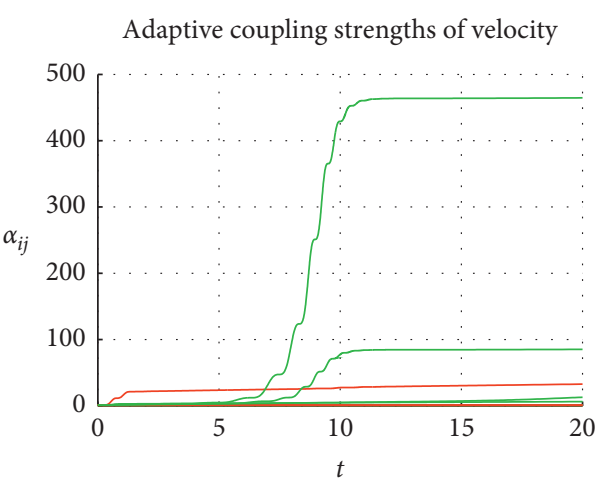

(f)

Adaptive feedback gain of velocity

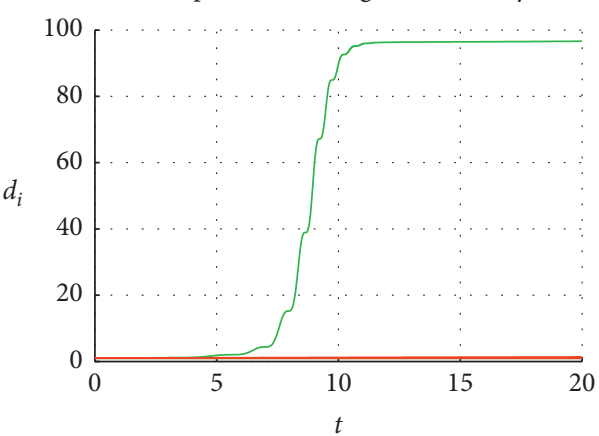

(h)

Figure 2: Intragroup coupling matrix is symmetric and time delay $\tau=0.1$. (a) Positions. (b) Velocities. (c) $c_{i j}$. (d) $d_{i j}$. (e) $c_{i}$. (f) $\alpha_{i j}$. (g) $\beta_{i j}$. (h) $d_{i}$.

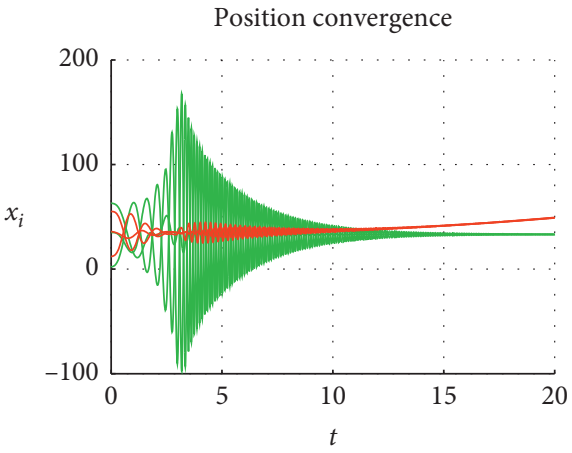

(a)

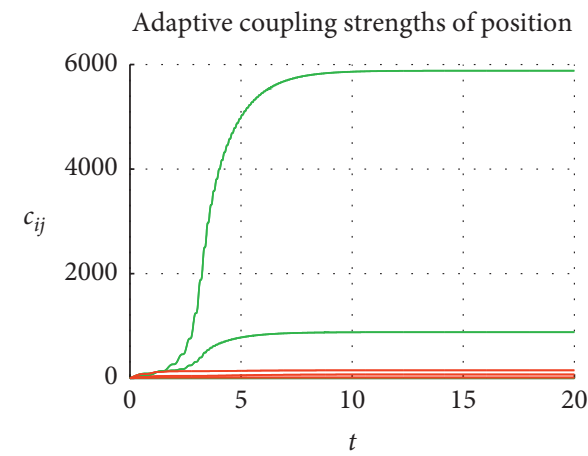

(c)

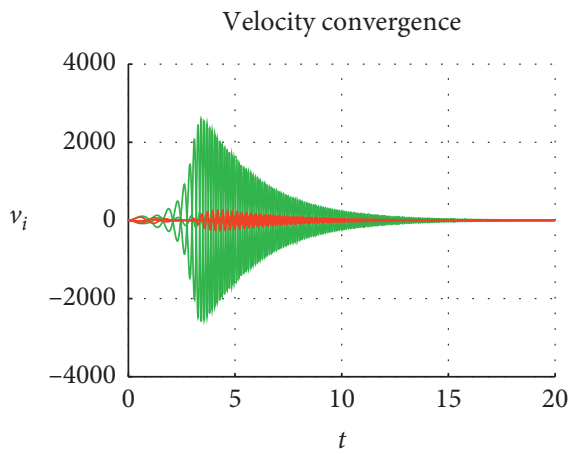

(b)

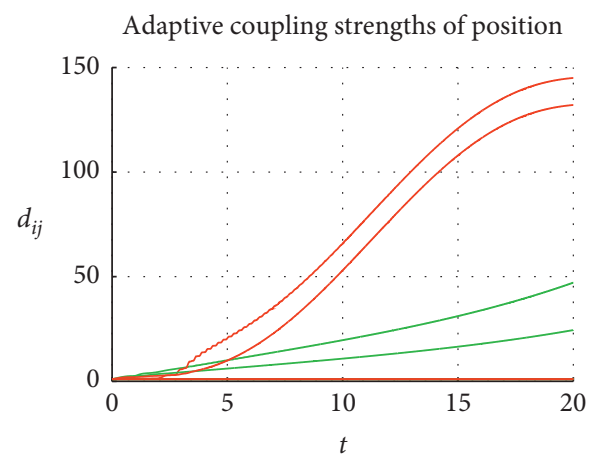

(d)

FIgUre 3: Continued. 


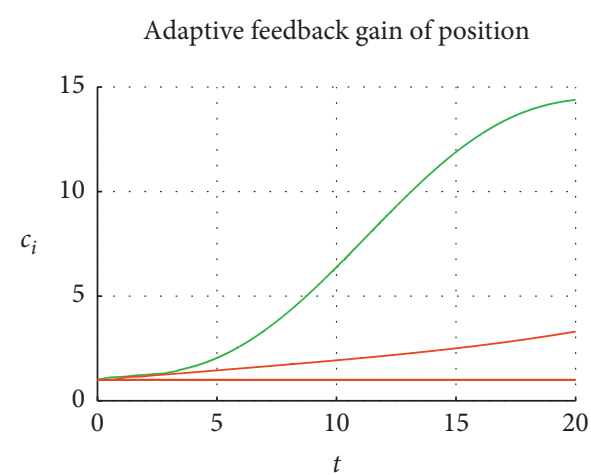

(e)

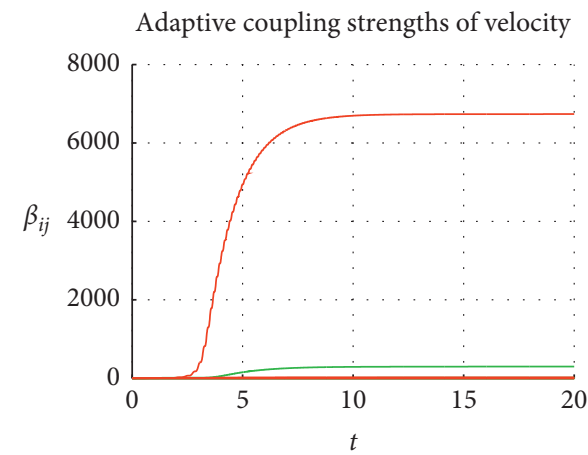

(g)

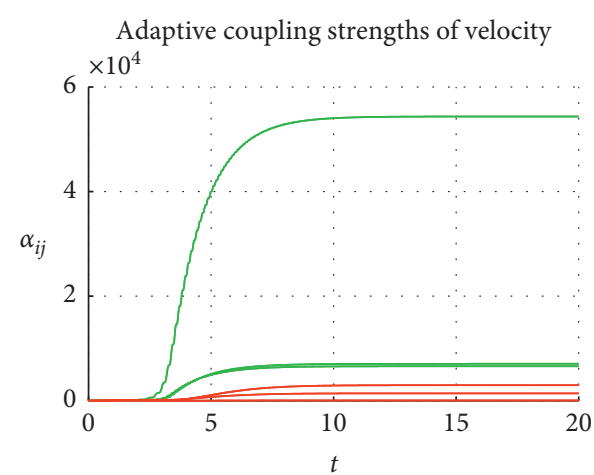

(f)

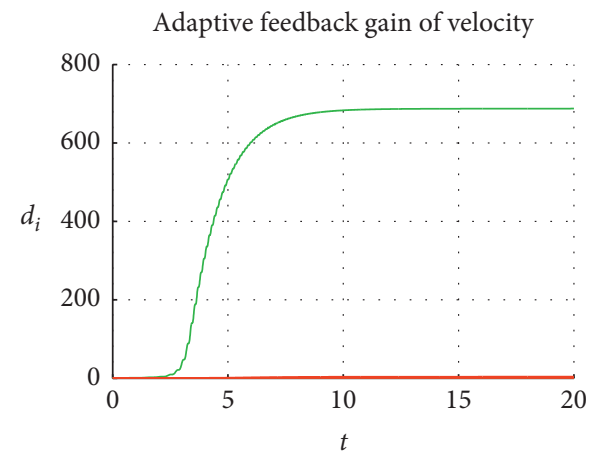

(h)

Figure 3: Intragroup coupling matrix is asymmetric and time delay $\tau=0.1$. (a) Positions. (b) Velocities. (c) $c_{i j}$. (d) $d_{i j}$. (e) $c_{i \cdot}$. (f) $\alpha_{i j}$. (g) $\beta_{i j}$. (h) $d_{i \text {. }}$
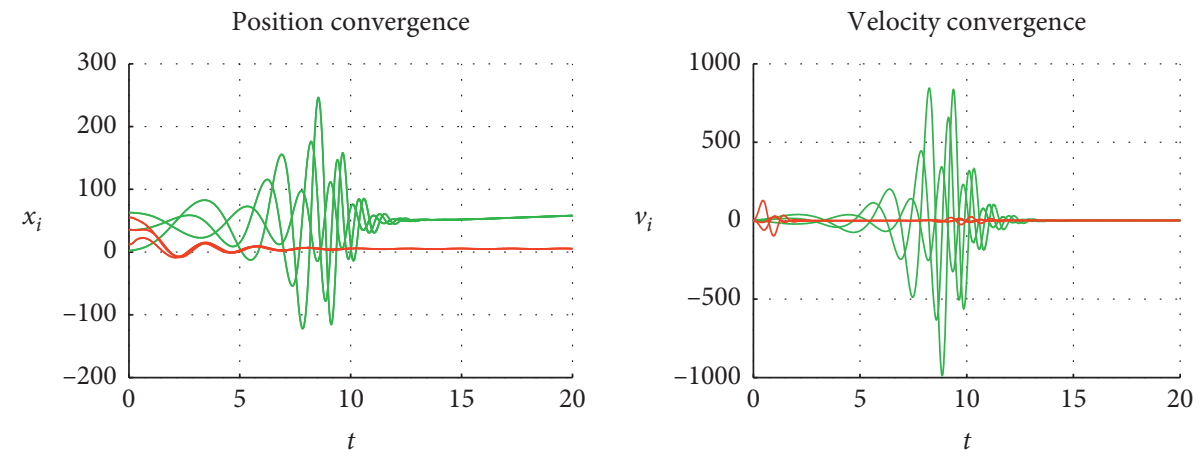

(a)
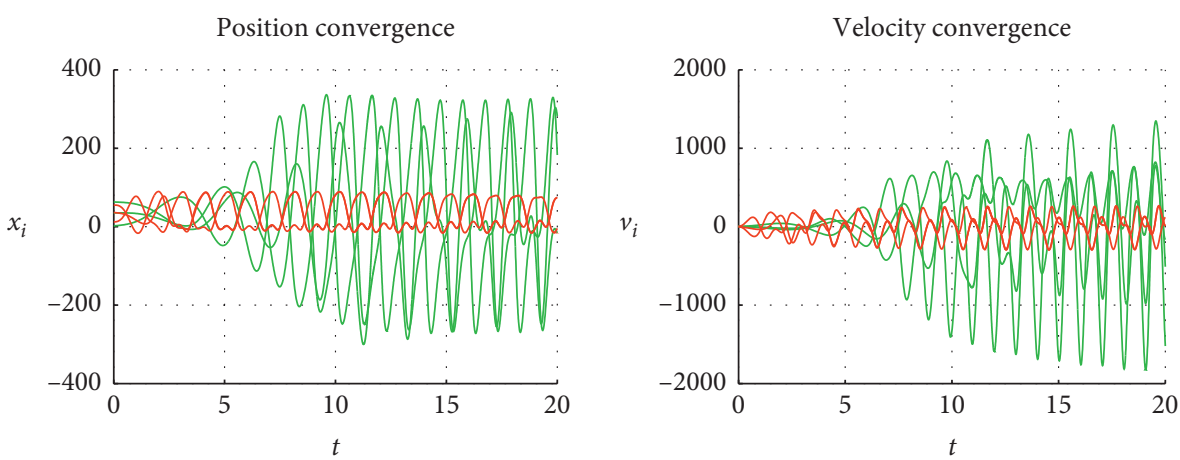

(b)

FIGURE 4: Intragroup coupling matrix is symmetric and with adaptive strategies. (a) Time delay $\tau=0.1$. (b) Time delay $\tau=1$. 


\section{Conclusion}

The adaptive group synchronization of second-order nonlinear complex dynamical networks with time-varying delays and sampled data has been researched in this paper. A new adaptive law has been designed, and we have proved that the second-order system with sampled data can achieve group synchronization no matter whether the coupling matrix is symmetric or not. Moreover, we have discussed the influences of time-varying delays and adaptive laws for group synchronization of complex networks with nonlinear dynamics in the different simulations. Finally, some simulations have been represented.

\section{Data Availability}

No data were used in this study.

\section{Conflicts of Interest}

The authors declare that there are no conflicts of interest regarding the publication of this paper.

\section{Acknowledgments}

This work was supported by the achievements of the important project "Class Teaching Experiment Research in Basic Education School" of Beijing Education Science "12th Five Year Plan" in 2015 (no. ABA15009) and National Natural Science Foundation of China (Grant no. 61773023).

\section{References}

[1] G. Chen and Z. Duan, "Network synchronizability analysis: a graph theoretic approach," Chaos: An Interdisciplinary Journal of Nonlinear Science, vol. 18, no. 3, Article ID 37102, 2008.

[2] C. Wu and L. Chua, "Application of graph theory to the synchronization in an array of coupled nonlinear oscillators," IEEE Transactions on Circuits and Systems I: Fundamental Theory and Applications, vol. 42, no. 8, pp. 494-497, 1995.

[3] A. Papachristodoulou, A. Jadbabaie, and U. Münz, "Effects of delay in multi-agent consensus and oscillator synchronization," IEEE Transactions on Automatic Control, vol. 55, no. 6 , pp. 1471-1477, 2010.

[4] X. L. Wang, H. Su, M. Z. Q. Chen, X. F. Wang, and G. Chen, "Reaching non-negative edge consensus of networked dynamical systems," IEEE Transactions on Cybernetics, vol. 48, no. 9, pp. 2712-2722, 2018.

[5] X. Liu, H. Su, and M. Z. Q. Chen, "A switching approach to designing finite-time synchronization controllers of coupled neural networks," IEEE Transactions on Neural Networks and Learning Systems, vol. 27, no. 2, pp. 471-482, 2016.

[6] B. Liu, N. Xu, H. Su, L. Wu, and J. Bai, "On the observability of leader-based multiagent systems with fixed topology," Complexity, vol. 2019, Article ID 9487574, 10 pages, 2019.

[7] C. Tang, B. Wu, J. Wang, and L. Xiang, "Evolutionary origin of asymptotically stable consensus," Scientific Reports, vol. 4, p. $4590,2014$.

[8] I. Z. Kiss, "Synchronization engineering," Current Opinion in Chemical Engineering, vol. 21, pp. 1-9, 2018.
[9] H. Su, Y. Sun, and Z. Zeng, "Semiglobal observer-based nonnegative edge consensus of networked systems with actuator saturation," IEEE Transactions on Cybernetics, vol. 50, no. 6, pp. 2827-2836, 2020.

[10] H. Su, Y. Liu, and Z. Zeng, "Second-order consensus for multiagent systems via intermittent sampled position data control," IEEE Transactions on Cybernetics, vol. 50, no. 5, pp. 2063-2072, 2020.

[11] H. Su, M. Long, and Z. Zeng, "Controllability of two-timescale discrete-time multiagent systems," IEEE Transactions on Cybernetics, vol. 50, no. 4, pp. 1440-1449, 2020.

[12] H. Su, J. Zhang, and X. Chen, "A stochastic sampling mechanism for time-varying formation of multiagent systems with multiple leaders and communication delays," IEEE Transactions on Neural Networks and Learning Systems, vol. 30, no. 12, pp. 3699-3707, 2019.

[13] S. Liu, Z. Ji, H. Ma, and S. He, "A new perspective to algebraic characterization on controllability of multiagent systems," Complexity, vol. 2020, Article ID 9703972, 12 pages, 2020.

[14] B. Liu, H. Su, L. Wu, X. Li, and X. Lu, "Fractional-order controllability of multi-agent systems with time-delay," Neurocomputing, 2020.

[15] X. Wang, H. Su, X. Wang, and G. Chen, "Fully distributed event-triggered semiglobal consensus of multi-agent systems with input saturation," IEEE Transactions on Industrial Electronics, vol. 64, no. 6, pp. 5055-5064, 2017.

[16] W. Yu, L. Zhou, X. Yu, J. Lu, and R. Lu, "Consensus in multiagent systems with second-order dynamics and sampled data," IEEE Transactions on Industrial Informatics, vol. 9, no. 4, pp. 2137-2146, 2013.

[17] B. Liu, P. Wei, and X. Wang, "Group synchronization of complex network with nonlinear dynamics via pinning control," in Proceeding of the 32nd Chinese Control Conference, IEEE, Xi'an, China, pp. 235-240, July 2013.

[18] W. Yu, G. Chen, and J. Lu, "On pinning synchronization of complex dynamical networks," Automatica, vol. 45, no. 2, pp. 429-435, 2009.

[19] Y. Lü, J. Lu, J. Liang, and J. Cao, "Pinning synchronization of nonlinear coupled Lure networks under hybrid impulses," IEEE Transactions on Circuits and Syatems II-Express Briefs, vol. 66, no. 3, pp. 432-436, 2018.

[20] H. Su, Z. Rong, M. Chen, X. Wang, G. Chen, and H. Wang, "Decentralized adaptive pinning control for cluster synchronization of complex dynamical networks," IEEE Transactions on Systems, Man and Cybernetics, Cybernetics, vol. 43, no. 1, pp. 394-399, 2013.

[21] P. Delellis, M. Bemnardo, and F. Garofalo, "Synchronization of complex networks through local adaptive coupling," Chaos, vol. 18 , no. 3, p. $175,2008$.

[22] J. Hu and W. X. Zheng, "Adaptive tracking control of leaderfollower systems with unknown dynamics and partial measurements," Automatica, vol. 50, no. 5, pp. 1416-1423, 2014.

[23] B. Liu, S. Li, and L. Wang, "Adaptive synchronization of two time-varying delay nonlinear coupled networks," in Proceedings of the 33rd Chinese Control Conference, pp. 38003804, IEEE, Nanjing, China, July 2014.

[24] Y. Zhao, G. Wen, Z. Duan, and G. Chen, "Adaptive consensus for multiple nonidentical matching nonlinear systems: an edge-based framework," IEEE Transactions on Circuits and Systems II: Express Briefs, vol. 62, no. 1, pp. 85-89, 2015.

[25] B. Liu, S. Li, and X. Tang, "Adaptive second-order synchronization of two heterogeneous nonlinear coupled networks," Mathematical Problems in Engineering, vol. 2015, Article ID 578539, 7 pages, 2015. 
[26] Q. Xu, S. Zhuang, Y. Zeng, and J. Xiao, "Decentralized adaptive strategies for synchronization of fractional-order complex networks," IEEE/CAA Journal of Automatica Sinica, vol. 4, no. 3, pp. 543-550, 2017.

[27] D. Ding, J. Yan, N. Wang, and D. Liang, "Adaptive synchronization of fractional order complex-variable dynamical networks via pinning control," Communications in Theoretical Physics, vol. 68, no. 9, pp. 366-374, 2017.

[28] L. Du, Y. Yang, and Y. Lei, "Synchronization in a fractionalorder dynamic network with uncertain parameters using an adaptive control strategy," Applied Mathematics and Mechanics, vol. 39, no. 3, pp. 353-364, 2018.

[29] S. Wang, H. Yao, S. Zheng, and Y. Xie, "A novel criterion for cluster synchronization of complex dynamical networks with coupling time-varying delays," Communications in Nonlinear Science and Numerical Simulation, vol. 17, no. 7, pp. 29973004, 2012.

[30] Z.-J. Tang, T.-Z. Huang, J.-L. Shao, and J.-P. Hu, "Consensus of second-order multi-agent systems with nonuniform timevarying delays," Neurocomputing, vol. 97, no. 1, pp. 410-414, 2012.

[31] B. Liu, X. Wang, H. Su, H. Zhou, Y. Shi, and R. Li, “Adaptive synchronization of complex dynamical networks with timevarying delays," Circuits, Systems, and Signal Processing, vol. 33, no. 4, pp. 1173-1188, 2014.

[32] Y. Liang and X. Wang, "Adaptive synchronization in complex networks through nonlinearly coupling," Computer Engineering and Applications, vol. 48, no. 10, pp. 25-28, 2012.

[33] H. Su, G. Chen, X. Wang, and Z. Lin, “Adaptive second-order consensus of networked mobile agents with nonlinear dynamics," Automatica, vol. 47, no. 2, pp. 368-375, 2011.

[34] B. Liu, X. Wang, Y. Gao, G. Xie, and H. Su, "Adaptive synchronization of complex dynamical networks governed by local lipschitz nonlinearlity on switching topology," Journal of Applied Mathematics, vol. 2013, Article ID 818242, 7 pages, 2013.

[35] H. Su, N. Zhang, M. Z. Chen, H. Wang, and X. Wang, "Adaptive flocking with a virtual leader of multiple agents governed by locally Lipschitz nonlinearity," Nonlinear Analysis: Real World Applications, vol. 23, no. 9, pp. 978-990, 2013.

[36] M. Li, B. Liu, Y. Zhu, L. Wang, and M. Zhou, "Group synchronization of nonlinear complex dynamics networks with sampled data," Mathematical Problems in Engineering, vol. 2014, Article ID 142061, 8 pages, 2014.

[37] H. Liu, G. Xie, and L. Wang, "Necessary and sufficient conditions for solving consensus problems of double-integrator dynamics via sampled control," International Journal of Robust and Nonlinear Control, vol. 20, no. 15, pp. 1706-1722, 2010.

[38] Y. Gao and L. Wang, "Sampled-data based consensus of continuous-time multi-agent systems with time-varying topology," IEEE Transactions on Automatic Control, vol. 56, no. 5, pp. 1226-1231, 2011.

[39] B. Liu, X. Wang, H. Su, Y. Gao, and L. Wang, "Adaptive second-order consensus of multi-agent systems with heterogeneous nonlinear dynamics and time-varying delays," Neurocomputing, vol. 118, pp. 289-300, 2013.

[40] J. Zhang, M. Li, and B. Liu, "Synchronization of a complex network for neighboring agents with time-varying delays," Communications in Computer and Information Science, vol. 225, pp. 73-81, 2011. 